\title{
Attitude on the concept of designing and analyzing experiments and its application in the engineering system
}

\begin{abstract}
Knowledge designing experiments is a powerful tool for engineers and managers who want to design or produce their products with high quality and low cost. As an irrefutable fact, the industry has recognized the increasing importance of quality. Today, quality is a business strategy for increasing market share, and organizations achieve global quality through experiments designed. Experiment design is one of the strongest techniques are improving quality and increasing productivity. In this way, by performing some tests, consciously changes are made to the process or system in order to examine their impact on the functional characteristics or response of the process or system to them. The design of experiments is the systematic manipulation of a number of variables in which the impact of these manipulations is evaluated and concluded, and the results are implemented. Test design and testing a set of techniques and statistical methods for collecting data from designed experiments, analysis, and, finally, conclusions and inferences about them. Several books have been written about theoretical issues and discuss how to design and analyze experiments. An analysis of a designed experiment usually requires a large number of numerical calculations that, as the number of data and variables examined increases, the complexity and timeliness of the problem are increased so that manual execution makes them impossible. Undoubtedly, the existence of many software packages in statistics, due to the need for and advancement in computer science, contributed greatly to the accuracy and convenience of data analysis.
\end{abstract}

Keywords: stest design, quality engineering, classical test design training, analyzing experiments, engineering system, design, theoretical issues, statistical control, data analysis, re-engineering
Volume 2 Issue 4 - 2018

\author{
Mojtaba Salehi \\ Department of Industrial Engineering, Payame Noor University \\ of North Tehran, Iran
}

\section{Correspondence: Saeid Mahmoudi, Department of Engineering, Payame Noor University of North Tehran, Iran, Email S.Mahmoudi44@yahoo.com}

Received: June 03, 2018 | Published: August 01, 2018

\section{Introduction}

Quality Issues In recent decades, new issues such as statistical control of the SPC process, overall quality management (TQM), reengineering (BPR), continuous Japanese improvement (Kaizen), quality cost analysis (COQ), . . Continuous improvement of the CPI process and so on.The Technological Design Test (DOE) is also a quality improvement technique that was introduced in the 1990s and 1980s as a competitive advantage in Western countries and Japan. The proper use of statistical testing techniques can facilitate the design and production of new products and improve existing products. These principles have been used in most industries such as electronics and semiconductors, aerospace, automobiles, medical equipment, food, pharmaceuticals, and chemical and process industries. It should be noted that the quality tools only have the ability to report defects and what factors and how they affect the quality of the process? How does the quality change if these factors change? How do these factors change to get the best quality performance? What are the controllable factors so that the effect of uncontrollable factors on quality is minimized? And ... all and all questions that can be answered by doing the test.

\section{What is the design test (DOE?)}

Researchers do experiments to discover phenomena to discover the truth about a system or process. Performing an experiment always involves cost and time. Therefore, performing effective experiments that earns the greatest amount of information with minimal cost and time is the aspiration of every engineer or researcher, and the cost and time when the number of factors increases ascending. Therefore, it is necessary in a way that can be achieved with the least amount of time and time, the most information about the process, provide logical conclusions and obtain documentary evidence of the process. The method best suited to meet the goals mentioned is the Design of Experiments (DOE). Also, the use of designing experiments helps engineers develop and evolve production, and processes that change against environmental factors and other resources. The timely and successful application of the design of the experiment in the development of the production process can essentially reduce production time and costs, and result in processes and products that, in their own form, have better performance and reliability than those utilized by other methods. ${ }^{1}$ By utilizing and understanding the design concept of experiments, managers and engineers find out how to bring competitive-quality products back to the international competition again with higher qualifications. Today's executives and engineers need to have enough information on design methods before deciding on the extent of product development and process development. That's why international companies such as Toyota announce many engineers annually under the DOE training course, and a company like Ford in the US, even its suppliers, is required to use this technique.According to the above, DOE technique can be used in most processes to improve quality. Processes in which their output has a qualitative attribute, a 
customer's request, or any other characteristic.In most industries, the correct and effective use of designing statistical tests is the key to increasing productivity, reducing variability, reducing latency in the design and development of better products and, therefore, customer satisfaction. The DOE technique is not limited to industrial and production environments. In all processes and systems, DOE can be used to increase output quality. In social systems where processes are human beings, in agriculture, which processes are plants, in economic systems that exchange processes, and ... DOE can be used to improve what we want from these systems. ${ }^{2}$

\section{Controlled variables}

In each experiment, a number of variables are provided to the analyst and they change them according to the testing needs they are doing. For this reason, these variables are also called controllable variables. Controlled factors are the factors that change by rotating a valve, changing a key, changing a piece, or increasing the amount of material. Uncontrollable variables create noise in the process and reduce the possibility of identifying controllable variables. Therefore, identification of these variables is also important and should be taken to collect information about them. One of the most important factors that cause failure in DOE is the lack of identification and collection of information related to these variables. Of the most common uncontrollable variables, changing the operator from one shift to another, changing the temperature of the environment and changing the raw materials that come from outside the company. Understanding the process requires practical experience and understanding of theoretical concepts in the hands of Hermes experts to ensure customers are fully aware of the process, variables and scope of their changes, and how they are controlled and measured. ${ }^{3}$ Using the techniques mentioned above, there may be a large number of input factors or variables. It is very difficult and even impossible to use all of them due to the large number of tests and measurement costs for each of them. Therefore, you must reduce the number of variables by selecting the most important ones. Hermes experts will be in the process of evaluation, selecting variables, and designing techniques, analyzing the results, and justifying process behavior and optimization alongside you.These analyzes are often performed by statistical analysis software, most notably Minitab and Design Expert. ${ }^{4}$

\section{The purpose of the design of the experiment (DOE)}

In fact, DOE's goal is to solve the following problem:

I. Earn maximum information and knowledge of processes

II. Minimal costs

III. Earn the minimum time to run the process

\section{Experimental design achievements (DOE):}

a. Understanding the characteristics of the product and process and determining the most effective and most important factors of process control

b. Determine how the process inputs (or control attributes) affect the output of the process and product (or qualitative or functional characteristics) in the shortest possible time and with the lowest possible cost.

c. Determine the input variables that have the most impact on the output of the product or process.

d. Modeling the process and determining the relationship between the inputs and outputs (product or performance) of the process and the action to continuously improve the product and process with the least cost and in the shortest time.

e. Optimizing and increasing the efficiency and efficiency of processes

f. Determine the exact value of the input parameters so that the output of the process is always close to the nominal value or target.

g. Determine the exact input parameters so that the output variability of the process is small.

h. Reducing the variability in the process involves determining the exact amount of controllable input parameters in such a way that the effect of the uncontrolled factors is minimized. Or, in other words, reducing the slope of process output (qualitative characteristics) sensitivity to factors that cannot be controlled and which increase waste and impair the output of the process (qualitative attributes). This is very useful for the uniform operation of the product under various environmental conditions.Determine the tolerance range or tolerance of process input factors (in scientific terms) such that the desired output is always within the permitted range.

i. Developing technical knowledge and recognizing product and process performance with respect to their input parameters and taking action to reduce the cost and time of developing and improving product and process.

\section{The Effects of DOE in developing the process:}
I. Improvement of efficiency
II. Reducing changes and adjusting to nominal values
III. Reduced development time
IV. Reducing costs

The Effects of DOE in engineering design activities:

a. Assess and compare the shape and dimensions of the design

b. Evaluation of materials

c. Determine the key parameters of product design that affect its function.

d. Optimal design of the product in the least time and with minimal cost

\section{Some common techniques in testing design topics include:}
I. Factor plans
II. Fractions Factor
III. Taguchi design
IV. RSM response procedure
V. Box design. ${ }^{6}$

\section{Test Design Applications (DOE)}

Understanding the characteristics of the product and the process and determining the most effective and important factors of process control: For example, in grinding a titanium aluminide alloy widely used in the next generation of aircraft and jet engines, the absence of surface damage such as cracks and tension in the surface is very important. . Accordingly, a project to study the effect of 5 parameters including the grinding parameters (shear rate and cutting depth) and the parameters of the grain (grain size, hardness, porosity) on the density and depth of the surface turquoise have been defined to determine which of the factors alone or in interaction with Other parameters are affected. The results of this study, which have been carried out with 
a limited number of tests, are virtually impossible to obtain without the use of experimental design techniques. For example, in many chemical processes, the percentage of materials in the mix to achieve desired output, such as viscosity, blinding power And so on. In such cases, there is no definite rule for obtaining the desired output formula (such as the power of cleansing) in terms of the composition of the raw materials, and the best way is to use the design techniques of the experiments to formulate the process, which then to reach any specific output value, The percentage of the combination of materials is easy to predict using the formula. Optimizing these kinds of cases, the engineering section seeks to find the best setting for the process so that the amount of each of the factors affecting the process is determined in such a way that one or more output parameters are optimized ${ }^{7}$ “Advising on Research Methods: A consultant's companion).

For example, in the Wirkkat machining process, it is possible to find the best input parameters in terms of both the machining speed and the best possible surface (free of imperfections). Optimization in the food industry: the problem of the lack of uniformity of the beverage level in the bottles. The uniformity of the beverage level is encountered in the bottle. Theoretically, the filling machine fills each bottle to the desired height, but in practice there is a deviation from the specified height. Manufacturers plan to detect these deviations by conducting experiments and reduce the level of fluid level as much as possible. Process engineers are able to control the three factors affecting the process: the percentage of carbon, filler pressure and the number of bottles produced (production speed) in Minutes Among the three factors above, the filling pressure and production speed are easily controllable, but the percentage of carbon during the actual process due to the variability of the temperature of the product is difficult to control. In solving this problem using DOE, performing only 24 experiments (with two replications And without much stoppage in the production line), the effectiveness of the factors has been determined and, after analyzing the results, the most suitable condition is the repeatability at the liquid heights inside the bottle, due to the oscillation of the work piece temperature, and the highest production speed is achieved, as the optimum state. If you do not use the design methods of experiments, not just the cost and the cost leaving one of the most serious potential issues in the final piece will lead to early turbine defeat. In order to investigate the effect of four factors of casting temperature, titanium content, type of heat treatment and grain refiner on the length of the cracks, the experiments were designed and analyzed using DOE. Application in manufacturing engineering and manufacturing. Example 1: Sensitivity of machining fineness to machine errors in machining. The blades used in the jet engine are powered by a five-axis $\mathrm{CNC}$ machine. The profile of the blade is one of the most important characteristics of the quality, in particular the degree of deformation of the blade profile compared to what has been specified in engineering drawings and computer models as reference profiles. In order to investigate the sensitivity of the blade profile deviation to possible errors in the machine, experiments have been carried out using DOE to determine the effect of changing each of the following factors on the blade profile deviation:

I. $0.2 \mathrm{~mm}$ deflection along the $\mathrm{X}$ axis

II. A bias of $0.2 \mathrm{~mm}$ in direction of $\mathrm{Y}$ axis

III. A bias of $0.2 \mathrm{~mm}$ in the direction of the $\mathrm{Z}$ axis

IV. Instrument seller (two types of tools)

V. Deviation of 0.001 degrees in the direction of the fourth axis

VI. 6. 10\%deviation in spindle speed
VII. $7.2 \mathrm{~mm}$ deviation in the height of the fixture

VIII. $10 \%$ deviation in forward speed. ${ }^{8}$

A methodology for designing experiments was proposed by Ronald Fisher, in his innovative books: The Arrangement of Field Experiments (1926) and The Design of Experiments (1935). Much of his pioneering work dealt with agricultural applications of statistical methods. As a mundane example, he described how to test the lady tasting tea hypothesis, that a certain lady could distinguish by flavor alone whether the milk or the tea was first placed in the cup. These methods have been broadly adapted in the physical and social sciences, are still used in agricultural engineering and differ from the design and analysis of computer experiments Simmons, Nelson and Simonsohn, 2011:1361)

\section{Comparison}

In some fields of study it is not possible to have independent measurements to a traceable metrology standard. Comparisons between treatments are much more valuable and are usually preferable, and often compared against a scientific control or traditional treatment that acts as baseline. ${ }^{9}$

\section{Randomization}

Random assignment is the process of assigning individuals at random to groups or to different groups in an experiment, so that each individual of the population has the same chance of becoming a participant in the study. The random assignment of individuals to groups (or conditions within a group) distinguishes a rigorous, "true" experiment from an observational study or "quasi-experiment" There is an extensive body of mathematical theory that explores the consequences of making the allocation of units to treatments by means of some random mechanism (such as tables of random numbers, or the use of randomization devices such as playing cards or dice). Assigning units to treatments at random tends to mitigate confounding, which makes effects due to factors other than the treatment to appear to result from the treatment.The risks associated with random allocation (such as having a serious imbalance in a key characteristic between a treatment group and a control group) are calculable and hence can be managed down to an acceptable level by using enough experimental units. However, if the population is divided into several subpopulations that somehow differ, and the research requires each subpopulation to be equal in size, stratified sampling can be used. In that way, the units in each subpopulation are randomized, but not the whole sample. The results of an experiment can be generalized reliably from the experimental units to a larger statistical population of units only if the experimental units are a random sample from the larger population; the probable error of such an extrapolation depends on the sample size, among other things. ${ }^{10}$

\section{Statistical replication}

Measurements are usually subject to variation and measurement uncertainty; thus they are repeated and full experiments are replicated to help identify the sources of variation, to better estimate the true effects of treatments, to further strengthen the experiment's reliability and validity, and to add to the existing knowledge of the topic. However, certain conditions must be met before the replication of the experiment is commenced: the original research question has been published in a peer-reviewed journal or widely cited, the researcher is independent of the original experiment, the researcher must first try to replicate the original findings using the original data, and the write-up 
should state that the study conducted is a replication study that tried to follow the original study as strictly as possible. ${ }^{11}$

\section{Blocking}

Blocking is the non-random arrangement of experimental units into groups (blocks/lots) consisting of units that are similar to one another. Blocking reduces known but irrelevant sources of variation between units and thus allows greater precision in the estimation of the source of variation under study.

\section{Example of orthogonal factorial design}

Orthogonality concerns the forms of comparison (contrasts) that can be legitimately and efficiently carried out. Contrasts can be represented by vectors and sets of orthogonal contrasts are uncorrelated and independently distributed if the data are normal. Because of this independence, each orthogonal treatment provides different information to the others. If there are $\mathrm{T}$ treatments and $\mathrm{T}-1$ orthogonal contrasts, all the information that can be captured from the experiment is obtainable from the set of contrasts.

\section{Factorial experiments}

Use of factorial experiments instead of the one-factor-at-a-time method. These are efficient at evaluating the effects and possible interactions of several factors (independent variables). Analysis of experiment design is built on the foundation of the analysis of variance, a collection of models that partition the observed variance into components, according to what factors the experiment must estimate or test. Discussion topics when setting up an experimental design. An experimental design or randomized clinical trial requires careful consideration of several factors before actually doing the experiment. An experimental design is the laying out of a detailed experimental plan in advance of doing the experiment. Some of the following topics have already been discussed in the principles of experimental design section:

How many factors does the design have, and are the levels of these factors fixed or random?

Are control conditions needed, and what should they be?

Manipulation checks; did the manipulation really work?

What are the background variables?

What is the sample size? How many units must be collected for the experiment to be generalisable and have enough power?

What is the relevance of interactions between factors?

What is the influence of delayed effects of substantive factors on outcomes?

How do response shifts affect self-report measures?

How feasible is repeated administration of the same measurement instruments to the same units at different occasions, with a posttest and follow-up tests?

What about using a proxy pretest?

Are there lurking variables?

Should the client/patient, researcher or even the analyst of the data be blind to conditions?

What is the feasibility of subsequent application of different conditions to the same units?

How many of each controls and noise factors should be taken into account?

The independent variable of a study often has many levels or different groups. In a true experiment, researchers can have an experimental group, which is where their intervention testing the hypothesis is implemented, and a control group, which has all the same element as the experimental group, without the interventional element. Thus, when everything else except for one intervention is held constant, researchers can certify with some certainty that this one element is what caused the observed change. In some instances, having a control group is not ethical. This is sometimes solved using two different experimental groups. In some cases, independent variables cannot be manipulated, for example when testing the difference between two groups who have a different disease, or testing the difference between genders (obviously variables that would be hard or unethical to assign participants to). In these cases, a quasiexperimental design may be used. ${ }^{12}$

\section{Causal attributions}

In the pure experimental design, the independent (predictor) variable is manipulated by the researcher - that is - every participant of the research is chosen randomly from the population, and each participant chosen is assigned randomly to conditions of the independent variable. Only when this is done is it possible to certify with high probability that the reason for the differences in the outcome variables are caused by the different conditions. Therefore, researchers should choose the experimental design over other design types whenever possible. However, the nature of the independent variable does not always allow for manipulation. In those cases, researchers must be aware of not certifying about causal attribution when their design doesn't allow for it. For example, in observational designs, participants are not assigned randomly to conditions, and so if there are differences found in outcome variables between conditions, it is likely that there is something other than the differences between the conditions that causes the differences in outcomes, that is - a third variable. The same goes for studies with correlational design.

\section{Statistical control}

It is best that a process be in reasonable statistical control prior to conducting designed experiments. When this is not possible, proper blocking, replication, and randomization allow for the careful conduct of designed experiments. To control for nuisance variables, researchers institute control checks as additional measures. Investigators should ensure that uncontrolled influences (e.g., source credibility perception) do not skew the findings of the study. A manipulation check is one example of a control check. Manipulation checks allow investigators to isolate the chief variables to strengthen support that these variables are operating as planned. One of the most important requirements of experimental research designs is the necessity of eliminating the effects of spurious, intervening, and antecedent variables. In the most basic model, cause $(\mathrm{X})$ leads to effect $(\mathrm{Y})$. But there could be a third variable $(\mathrm{Z})$ that influences $(\mathrm{Y})$, and $\mathrm{X}$ might not be the true cause at all. $\mathrm{Z}$ is said to be a spurious variable and must be controlled for. The same is true for intervening variables (a variable in between the supposed cause $(\mathrm{X})$ and the effect $(\mathrm{Y})$ ), and anteceding variables (a variable prior to the supposed cause (X) that is the true cause). When a third variable is involved and has not been controlled for, the relation is said to be a zero order relationship. In most practical applications of experimental research designs there are several causes (X1, X2, X3). In most designs, only one of these causes is manipulated at a time.

\section{Experimental designs after fisher}

Some efficient designs for estimating several main effects were found independently and in near succession by Raj Chandra Bose and K. Kishen in 1940 at the Indian Statistical Institute, but remained 
little known until the Plackett-Burman designs were published in Biometrika in 1946. About the same time, C. R. Rao introduced the concepts of orthogonal arrays as experimental designs. This concept played a central role in the development of Taguchi methods by Genichi Taguchi, which took place during his visit to Indian Statistical Institute in early 1950s. His methods were successfully applied and adopted by Japanese and Indian industries and subsequently were also embraced by US industry albeit with some reservations.In 1950, Gertrude Mary Cox and William Gemmell Cochran published the book Experimental Designs, which became the major reference work on the design of experiments for statisticians for years afterward. Developments of the theory of linear models have encompassed and surpassed the cases that concerned early writers. Today, the theory rests on advanced topics in linear algebra, algebra and combinatorics. As with other branches of statistics, experimental design is pursued using both frequentist and Bayesian approaches: In evaluating statistical procedures like experimental designs, frequentist statistics studies the sampling distribution while Bayesian statistics updates a probability distribution on the parameter space. Some important contributors to the field of experimental designs are C. S. Peirce, R. A. Fisher, F. Yates, C. R. Rao, R. C. Bose, J. N. Srivastava, Shrikhande S. S., D. Raghavarao, W. G. Cochran, O. Kempthorne, W. T. Federer, V. V. Fedorov, A. S. Hedayat, J. A. Nelder, R. A. Bailey, J. Kiefer, W. J. Studden, A. Pázman, F. Pukelsheim, D. R. Cox, H. P. Wynn, A. C. Atkinson, G. E. P. Box and G. Taguchi. [Citation needed] The textbooks of D. Montgomery, R. Myers, and G. Box/W. Hunter/J.S. Hunter have reached generations of students and practitioners. ${ }^{13}$

\section{Conclusion}

Test design techniques can significantly improve the design and development of products and related problem-solving activities. In today's world, manufacturers have to update their techniques and techniques to survive and continue their presence in the markets. Specifically, control and quality control techniques are important in this regard. The Technological Design Test (DOE) is one of the quality improvement techniques that was introduced in the 1990s and 1980s as a competitive advantage in Western countries and Japan, as one of the QS 9000 certification requirements. In our country, in recent years, steps have also been taken to implement DOE. It should be noted that the quality tools are only capable of reporting flaws and failures and that their users can use the correct way to improve the quality of the processes under Control yourself. If the purpose of using these tools is to be forgotten and the tools themselves are considered as goals, not only will quality improvements not be seen, but the lack of impact of these tools will cause dissatisfaction and serious risks to process management. When it comes to DOE, it's meant to do a research, but a specific research. A research is an activity that creates more awareness for a researcher. Research is in fact a kind of curiosity. Curiosity related to finding the truth. There are usually two types of research. In the first instance, the researcher attempts to create concepts and prove science, and thus provide a method. In the second type, research is applied. In applied research, the researcher attempts to answer a specific question. So in such cases, the researcher has a hypothesis trying to test it.In fact, the DOE includes a systematic program for conducting surveys conducted using statistical techniques. In these tests, factors or factors that are involved in a process are used in different situations, and information is collected from the final state of the product or its effect on the final product or process.

\section{Acknowledgements}

None.

\section{Conflict of interest}

The author declares there is no conflict of interest.

\section{References}

1. Sun, Tao, A transformable wheel-legged mobile robot: Design, analysis and experiment, Journal of Robotics and Autonomous Systems. 2017:98:30-41.

2. Nowzari, Raheleh, Mirzaei. Finding the best configuration for a solar air heater by design and analysis of experiment, Journal of Energy Conversion and Management. 2015;100:131-137.

3. Bailey RA, Reiss, Julia. Design and analysis of experiments testing for biodiversity effects in ecology. Journal of Statistical Planning and Inference. 2014;144:69-80.

4. Zea, Luis, Zeena Nisar, Phil Rubin, et al. Design of a spaceflight biofilm experiment, Journal of Acta Astronautica. 2018;19:17-29.

5. Cohen, Achraf. Design of experiments and statistical process control using wavelets analysis, Journal of Control Engineering Practice. 2016;49:129-138.

6. Wannes GM. Optimal design of experiments for excipient compatibility studies, Journal of Chemometrics and Intelligent Laboratory Systems. 2017;171:125-139.

7. Ader, Mellenberg, Hand P. Advising on Research Methods: A consultant's companion. 2008

8. Anderson, Christine. Design of experiments and data analysis challenges in calibration for forensics applications. Journal of Chemometrics and Intelligent Laboratory Systems. 2015;149:107-117.

9. Myers, Raymond. Generalized linear models: with applications in engineering and the sciences. 2010.

10. Creswell JW. Educational research: Planning, conducting, and evaluating quantitative and qualitative research 3 rd edition. Upper Saddle River, NJ: Prentice Hall; 2008.p. 1-673.

11. Burman Leonard E, Robert W Reed, James Alm. A call for replication studies. Public Finance Review. 2010;38(6):787-793.

12. Zacks S. Adaptive Designs for Parametric Models. In: Ghosh S, et al editors. Design and Analysis of Experiments, Handbook of Statistics. Volume 13. North-Holland. ISBN 0-444-82061-2. 1996. p. 151-180.

13. Simmons, Joseph, Leif Nelson, et al. False-Positive Psychology: Undisclosed Flexibility in Data Collection and Analysis Allows Presenting Anything as Significant. Psychol Sci. 2011;22(11):13591366. 